\title{
INNOVACIÓN PÚBLICA PARA LA SEGURIDAD: EL CASO NUEVO LEÓN, MÉXICO
}

\author{
Juan Carlos Montero \\ juancarlosmonterob@itesm.mx \\ Instituto Tecnológico y de Estudios Superiores de Monterrey, Campus \\ Monterrey, México
}

\section{RESUMEN}

Entre 2009 y 2011, Nuevo León y la región noreste de México sufrieron su peor crisis de seguridad. Surgieron surgiendo fuertes críticas y presiones hacia el gobierno del estado para cambiar su estrategia. El artículo, a partir de un análisis documental y la aplicación de entrevistas a actores clave, se analizan las modificaciones en materia de seguridad que se implementaron como respuesta a la crisis. En especial, el artículo estudia la transformación de la red de política pública, la creación de la nueva policía estatal y la aparición de una nueva forma de relación entre sociedad y gobierno para la atención del tema de desapariciones forzadas en Nuevo León. El artículo muestra que las políticas de seguridad no es un asunto exclusivo de las policías sino requiere el involucramiento de la sociedad civil en las etapas de diseño e implementación.

Palabras clave: Innovación, Políticas públicas, Seguridad ciudadana, México. 


\title{
PUBLIC INNOVATION FOR SECURITY: THE CASE OF NUEVO LEÓN, MEXICO
}

\begin{abstract}
Between 2009 and 2011, Nuevo León and the north-east region of Mexico suffered their worst security crisis. Harsh criticism and pressure emerged, directed at the state government to change its strategy. This article, using documentary analysis and interviews with key players, analyzes the modifications in security that were implemented as a response to the crisis. Specifically, the article studies the transformation of the public policy network, the creation of the new State police and the appearance of a new type of relationship between society and government to attend to the matter of the forced disappearances in Nuevo León. The article shows that the security policies are not the sole domain of the police, but rather that they require the involvement of civil society in the design and implementation stages.
\end{abstract}

Keywords: Innovation, Public policies, Citizen security, Mexico. 


\section{INTRODUCCIÓN}

En el contexto de la guerra contra las organizaciones criminales en México, específicamente entre los años 2009 y 2010, el noreste del país enfrentó la peor crisis de seguridad en su historia. Grupos del crimen organizado se enfrentaron violentamente en los estados de Nuevo León, Tamaulipas, Coahuila y San Luis Potosí. Entre los cuatro estados que integran la región, Nuevo León, un estado tradicionalmente ajeno a la violencia en México, sufrió el incremento más grave en el número de homicidios, pero a través de un ejercicio de innovación pública transformó la política en materia de seguridad, contribuyendo a reducir los homicidios a un ritmo mayor que el resto.

La innovación se expresó en el desarrollo de nuevas instituciones policiales y una nueva forma de relacionarse entre sociedad y gobierno. El proceso transformó las relaciones de gobernanza en la red de la política de seguridad, pasando de monopolios o triángulos de hierro a redes más amplias con la participación de diversos actores; el resultado, implicó la creación de la nueva policía estatal Fuerza Civil, en cuyo diseño participaron universidades y el sector empresarial local, configurando la tradicional triple hélice para la innovación (Etzkowitz, y Leydesdorff 2000); por otro lado, a partir de la presión de organizaciones no gubernamentales, se transformó la atención a víctimas, especialmente relacionadas con desapariciones forzadas, ejecuciones extrajudiciales y otras violaciones a los derechos humanos.

La relevancia del caso de Nuevo León estriba en las siguientes cuestiones. En primer lugar, la nueva policía estatal ha sido reconocida por los presidentes de la república Felipe Calderón y Enrique Peña Nieto, como el ejemplo a seguir por el resto de los estados; en esa dirección, estados como Coahuila, México, Michoacán, Tamaulipas, Veracruz, han buscado replicar el caso. En segundo lugar, el tema de las desapariciones forzadas ha tomado una gran relevancia en México, pero ha sido poco o nada atendido por el gobierno; sin embargo, en el periodo de estudio se inauguró una nueva forma de relación entre el gobierno del estado, organizaciones defensoras de los derechos humanos y víctimas.

El objetivo del trabajo es exponer la transformación de la política de seguridad en Nuevo León en el periodo 2009-2015, a través de la innovación pública como respuesta a la crisis de seguridad vivida entre los años 2009-2011. En esta dirección, a lo largo de este artículo se analizar la creación de la nueva policía estatal y la nueva forma de relacionarse entre sociedad y gobierno para la atención del tema de desapariciones forzadas en Nuevo León. Para ello, en primer lugar se expondrán los conceptos fundamentales para definir la innovación, innovación gubernamental e 
innovación pública; en segundo lugar, se expondrá el caso de Nuevo León $y$; finalmente, se presentan las conclusiones.

\section{INNOVACIÓN, INNOVACIÓN PÚBLICA Y POLÍTICAS PÚBLICAS}

La definición del concepto innovación es difusa, pero se reconoce como determinante para la competitividad de organizaciones, ciudades y regiones. La construcción de medios para generarla tampoco son claros y han derivado en la generación de una amplia literatura para tratar de acercarse a la definición del concepto; en este sentido, la innovación se ha convertido en un concepto con significado poco claro, pero que al mismo tiempo es obsesión para competir (Köhler y González 2014: 69). El concepto se define como la creación, implantación ${ }^{1}$ y adopción ${ }^{2}$ de nuevos comportamientos o ideas en una organización, los cuales se refieren a nuevos productos, servicios, tecnologías, prácticas administrativas o hallazgos de investigación ${ }^{3}$ que rompen con paradigmas vigentes (Hage

1 La implantación implica “[...] poner en ejecución los elementos para concretar una acción múltiple (instituciones, doctrinas, prácticas o costumbres” (Arellano 2004).

2 Entendemos por adopción el compromiso de los integrantes de una organización hacia la incorporación de nuevos productos o procesos definidos como innovadores. En sentido contrario, se puede decir que la innovación no se adopta cuando los integrantes de la organización no asumen como propias las innovaciones y las rechazan o no se comprometen con ellas (Klein y Speer 1996, Hage 2005, Damanpour y Schneider 2009). La importancia de la adopción radica en que constituye un cambio institucional; es decir, cambios que conduzcan a un nuevo orden con nuevas reglas (escritas y no escritas), valores, incentivos y sanciones que permitan a la organización adaptarse mejor a su entorno y alcanzar sus objetivos (Ostrom 2008: 151); implica -sobre todo al tratarse de productos o servicios- la capacidad de incorporarse al mercado y constituirse como una ventaja (García y Calantone 2002: 112), lo cual requiere compromiso e involucramiento (engagement) por parte de los integrantes de la organización y aceptación por parte de los actores que integran el contexto; en otras palabras, la innovación no implica únicamente nuevos productos o servicios sino también una forma diferente de hacer las cosas en la organización, cambios en valores y creencias de sus integrantes, todo ello alineado de forma metodológica y sistemática (Teece 2010: 186, Keeley 2013: 26).

3 Es necesario diferenciar la innovación como producto y como proceso. Como producto, puede entenderse como un nuevo conocimiento o una nueva idea, generalmente producto de investigación. Como proceso, la innovación es consecuencia de una cadena de valor que vincula el conocimiento con el mercado y que implica la difusión o comercialización de un producto, por ejemplo a través de traducir una investigación en patente y aplicarla a un sector económico (Morgan 
2005: 71, Keeley 2013: 5). A partir de la magnitud de la ruptura y su impacto en la organización que los realiza, se habla de innovación radical/ discontinua; continua/incremental; reinnovación; entre otros (García y Calantone 2002: 117).

La innovación es producto de la acción colectiva e implica una cadena que relaciona el lugar en el que ocurre, las organizaciones que la conducen, los individuos que generan las ideas y el conocimiento, así como el contexto en donde se generan los incentivos o inhibidores; en palabras de Köhler y González (2014: 69) "la fuerza competitiva que deriva de la innovación no reside únicamente en los conocimientos individuales, sino más bien en su concentración y combinación eficaz en forma de conocimientos colectivos"; en consecuencia, la innovación es un producto social y no es exclusiva de empresas u otras organizaciones formales, sino también de grupos informales que se asocian entre sí para la obtención de un bien público a través de la acción colectiva.

La innovación depende de la capacidad de las organizaciones, grupos o comunidades para estructurarse en torno a una cadena, una estructura organizacional o modelo de negocio, que vincule la generación de conocimiento con el contexto, con la finalidad de generar valor; en consecuencia, no cualquier idea o cambio se constituye como innovación, solo aquellos que generen valor. El valor generado implica no solo utilidades sino también crecimiento económico y bienestar para los habitantes de las ciudades o regiones donde se genera, creando condiciones para la atracción de nuevos talentos y nuevas organizaciones emprendedoras que retroalimentan y fortalecen la dinámica de innovación (Florida 2011: 21, Scheel y Pineda 2015: 23, Villarreal y Flores 2015: 119). Cuando la innovación no logra adoptarse, tanto al interior de la organización como en su contexto, no se logra la generación de valor e incluso pueden generarse desequilibrios, tanto internos como externos, los productos y servicios se vuelven obsoletos y la organización desperdicia sus recursos, consideración que es fundamental al tratarse de gobiernos, cuyas políticas muchas veces son de corto alcance y tienden a ser obsoletas rápidamente (Scheel y Pineda 2015: 32).

La capacidad innovadora de la organización no solo depende de su dinámica interna sino también del contexto. Para sobrevivir y competir, las organizaciones buscan adaptarse a contextos complejos y cambiantes, donde se generan los incentivos o inhibidores para la innovación (Köhler y González 2014: 69). Si las organizaciones se insertan en un contexto que no

1997: 480-481, Casper y Van Waarden 2005: 10). 
cambia o que no permite vincular a las organizaciones con la generación de conocimiento, entonces los procesos organizacionales tampoco cambian y no se puede generar la innovación; si por el contrario, las organizaciones tienen incentivos para vincularse con otros individuos y organizaciones para la generación de conocimiento, vincular sus recursos para transformar dichos conocimientos en nuevos productos, así como mecanismos para colocar estos productos en el mercado global, se generan condiciones adecuadas para innovar.

$\mathrm{Al}$ asumir que los contextos son cambiantes, la innovación tiene un periodo de vida y tiende a ser obsoleta, por lo que las organizaciones requieren hacer de la innovación un proceso sistemático, que les permita adaptarse y aprovechar el contexto de manera constante. Para que esto suceda, se requiere de un modelo, sistema o metodología que genere incentivos para la atracción y vinculación de los recursos necesarios para: investigación y desarrollo; implementación; adopción al interior de la organización; colocación y adopción de los nuevos productos o servicios en el contexto (Klein y Speer 1996, Cooke, Gómez y Etxebarría 1997, Hage 2005, Roper, Du y Love 2008). Adicionalmente, requiere de agentes o administradores que, bajo un enfoque estratégico de largo plazo, la promuevan y conduzcan, con el objetivo de asegurar la canalización de recursos necesarios para la investigación, su aprovechamiento e implantación en la organización a través de la transformación en la estructura, así como en el sistema de creencias y valores, la cual constituye una figura equivalente a la del altruista en términos de acción colectiva o del emprendedor de políticas, en términos del análisis de política pública (Kingdon 1984, Grady 1992, Kano 2005, Arellano 2004, Ostrom 2008, Damanpour y Schneider 2009, Mariñez 2015).

El valor que se genera a partir de la innovación no beneficia solo a las organizaciones sino que también se traduce en bienestar. En ese sentido, la innovación se convierte en un elemento perseguido no solo por las organizaciones sino también por ciudades y regiones, teniendo por objetivo construir el entorno que requieren las organizaciones para incentivar la innovación. Dicho entorno, denominado ecosistema de innovación o sistema de innovación, tiene como objetivo el que las ideas generadas a partir de la investigación (sobre todo la realizada en universidades, pero también en otras organizaciones), cuenten con incentivos para permitir que el conocimiento se convierta en impulsor no solo de la organización sino también de ciudades y regiones (Etzkowitz y Leydesdorff 2000, Meeus y Oerlemans 2005, de la Mothe 2006, Florida 2011, Scheel 2012), las cuales compiten globalmente para atraer los recursos necesarios para construir sus sistemas de innovación; en palabras de Scheel y Pineda (2015: 23), 
[...] si se quiere hablar de innovación y de su impacto holístico regional, se debe articular la compleja dependencia de la innovación con la estructura política, con las regulaciones ecológicas, con el capital social y con el capital natural de las regiones [...].

En consecuencia, la innovación es, sobre todo, cooperación; sin la suma de esfuerzos, no se puede innovar y para ello, se requiere que el gobierno asuma el papel de agente promotor de la innovación y que, a través de una gestión estratégica, implemente políticas públicas que generen los incentivos para la atracción de talento, estableciendo las condiciones necesarias para el desarrollo de la cadena de innovación, especialmente para la cooperación, vinculando individuos y organizaciones entre sí y con el mercado ${ }^{4}$ (Florida 2011). A través de una adecuada estrategia gubernamental y apoyándose en la implementación de políticas educativas, de desarrollo urbano, desarrollo social, económicas, las ciudades buscan desarrollar sus capacidades para ser competitivas globalmente y atraer empresas, organizaciones e individuos que retroalimenten e impulsen la innovación, impulsando su economía, promoviendo el desarrollo humano, volviéndolas más sólidas y resilientes ante los vaivenes de la economía (de la Mothe 2006, Florida 2011, Scheel 2012).

Además de reconocer el papel del gobierno como agente promotor de la innovación mediante el desarrollo de sistemas de innovación, también se le debe reconocer como una organización más. Sin embargo, es una organización con recursos especiales: poder para la aplicación de la ley y gasto público (Downs 1957), pero que al mismo tiempo son insuficientes para atender todas las necesidades y demandas sociales, lo que obliga a tratar de maximizar sus recursos y a reconocer que el contexto en el que se ubica se configura como una compleja red $^{5}$ con la participación de diferentes actores, con diferentes intereses y recursos, donde empresas y gobierno

4 La construcción de los sistemas se apoya de políticas que promuevan la aglomeración de empresas vinculadas entre sí, la formación de clústers, con la finalidad de generar cadenas productivas y economías a escala (Carrillo 2015, Villarreal y Flores 2015); adicionalmente, implica políticas para que la investigación generada en las universidades no sirva solo a las empresas o el gobierno sino que también tengan un impacto social, lo que difícilmente sucedería si la investigación fuera desarrollada por las organizaciones para sí mismas (Hane 1999: 55).

5 Entendemos por red a una forma de organización informal entre jerarquías y mercados, cuyo objetivo es el intercambio de conocimiento, información, tecnología y valor. Su estructura es informal, sus límites son difíciles de establecer y los actores entran y salen de la red buscando sus diversos intereses particulares (de la Mothe 2002: 255). 
solo son uno más (Arellano 2004, Moore y Hartley 2008, del Castillo 2007, Mariñez 2015). Sin embargo, los recursos del gobierno generan una competencia a su alrededor para influir la toma de decisiones para el ejercicio de dichos recursos en torno a los intereses particulares de los actores (Sabatier 2007, del Castillo 2007, Tsebellis 2006), especialmente para la integración de las agendas de gobierno y los procesos de toma de decisiones (Kingdon 1984, Elder y Cobb 2000), articulándose a través de instituciones ${ }^{6}$, formales e informales, que configuran la gobernanza de la red de actores (Arellano 2004, Howlett 2009, Howlett y Ramesh 2003, Carter 2000).

El reconocimiento de que el gobierno se inserta en una compleja red en donde solo es un actor más, implica aceptar que la cooperación y la innovación ocurren entre diferentes actores con y sin la participación del gobierno. Los actores se relacionan entre sí, comunicándose e intercambiando recursos para resolver sus problemas a través de la innovación social entendida como "los cambios en las instituciones de gobernanza que buscan tener el efecto de contribuir a mejorar la calidad de vida en un contexto socialmente inclusivo y más justo" (González y Healey 2005: 2052), donde el gobierno puede o o no involucrarse en dichos cambios, rompiendo con la idea de que los problemas públicos requieren necesariamente de la intervención del gobierno y obligando a replantear los límites entre lo público y lo privado (Ostrom 2000, Cabrero 2003, Swyngedouw 2005, Gerometta, Häussermann y Longo 2005).

$\mathrm{Al}$ asumir al gobierno como un actor organizacional se debe reconocer su capacidad para innovar de manera similar a cualquier otra organización, pero también se debe analizar su relación con el contexto, la cual es muy diferente a la que establecen otras organizaciones como las empresas. Para analizar los procesos al interior del gobierno, nos apoyaremos del concepto de innovación gubernamental, el cual entendemos como el

6 Las instituciones, entendidas básicamente como las reglas del juego, determinan quién y cómo participa, las recompensas y los castigos, quién regula, premia o castiga y quién toma las decisiones; son aceptadas bajo la consideración de que el beneficio esperado por su participación es mayor que el no acatarlas. Su origen y finalidad es la resolución de problemas colectivos su continuidad y estabilidad se funda en la aceptación que tengan los individuos de las reglas establecidas (legitimidad), la adaptación de la institución a los valores de los actores que la influyen y la adaptación de la institución al entorno. La incapacidad de adaptación de las instituciones deriva en pérdida de legitimidad, crisis y desaparición de las mismas, lo cual constituye la necesidad de innovación (North 1990, Ostrom 2000). Las instituciones catalizan o inhiben la innovación, promueven la cooperación, la formación de alianzas y la creación de cadenas de innovación (de la Mothe 2006: 94). 
desarrollo de "[...] estructuras y formas organizativas no convencionales, formas novedosas de organización del trabajo, estructuras organizacionales no jerárquicas y programas de cambio planeado [...]" (Cabrero, Arellano y Amaya 2007: 148), que se expresan en políticas públicas innovadoras. El concepto implica una transformación en la forma de colaborar al interior del gobierno (relaciones intragubernamentales) y entre órdenes de gobierno (relaciones intergubernamentales), con la finalidad de maximizar sus recursos y brindar mejores resultados, en un contexto donde la cooperación se podría considerar como obvia, pero que en realidad suele ser de competencia, lo cual se expresa en innovación en los servicios gubernamentales, nuevas formas de relacionarse con los ciudadanos, nuevas estructuras organizacionales y políticas públicas innovadoras (Cabrero, Arellano y Amaya 2007, Bloch y Bugge 2013, Mariñez 2015). Debemos recordar que la innovación no es un evento excepcional sino que implica la transformación organizacional para generarla de manera sistemática, por lo que la innovación gubernamental requiere de una gestión estratégica; las políticas que no formen parte de la estrategia tenderán a ser obsoletas rápidamente y sus resultados podrían constituir un desperdicio de recursos ${ }^{7}$.

La principal diferencia en el contexto en que se inserta el gobierno es la política, entendida como la lucha entre actores para influir al gobierno. Sin embargo, al señalar anteriormente que la red de actores considera al gobierno solo como un actor más y que la cooperación para la atención de problemas públicas se construye con y sin la participación del gobierno, se llega al reconocimiento de nuevos esquemas de colaboración entre sociedad y gobierno que han permitido fortalecer los mecanismos de participación democrática, establecer nuevas relaciones en torno a los procesos de política pública, especialmente alrededor de la toma de decisiones y rendición de cuentas, los cuales han contribuido a fortalecer las instituciones políticas (Girouard y Sirianni 2014). En consecuencia, al reconocer que la resolución de los problemas públicos no solo es consecuencia de la intervención del gobierno, requerimos de un concepto que se enfoque no solo en los procesos al interior del gobierno sino en la interacción y cooperación del

7 La importancia de la gestión estratégica radica en la capacidad de articular el conjunto de recursos hacia un objetivo común como sociedad (Arellano 2004), lo que permitiría hablar de ciudades, regiones o países innovadores.. Sin lugar a dudas este elemento eleva significativamente la complejidad de la gestión, pero sus resultados determinan la competitividad, resiliencia y prosperidad, tal como han demostrado autores como Florida (2011) o Scheel y Pineda (2015), entre muchos otros estudios. Ante la dificultad de este tipo de esfuerzos, en vez de gestiones estratégicas existen múltiples casos de políticas públicas innovadoras, pero que al no tener trascendencia, no generan el valor esperado y se vuelven obsoletas. 
gobierno con otros actores.

La innovación generada a partir de las relaciones más allá de lo gubernamental, constituye la característica principal de la innovación pública. Este tipo de innovación implica transformaciones de las interacciones entre actores (Swyngedouw 2005, Mariñez 2015); especialmente entre el gobierno y la sociedad, el gobierno y otros órdenes de gobierno; todo ello, con el objetivo de generar valor público; en otras palabras, implica transformaciones en la gobernanza ${ }^{8}$ hacia un conjunto de instituciones que promuevan la cooperación, la comunicación de manera horizontal, el compromiso cívico, la transparencia y la rendición de cuentas (Moore y Hartley 2008: 4, Marińez 2015: 84). Estas nuevas relaciones entre actores que no están acostumbrados a trabajar juntos, contribuye a la formación de nuevas ideas y nuevas formas de resolver problemas, conduciendo a la generación de valor público; en palabras de Mariñez (2015: 25), la innovación pública “[...] apuesta a una relación de colaboración entre actores gubernamentales y no gubernamentales para el impulso de decisiones y políticas públicas que logren el mejoramiento de los servicios públicos con nuevas soluciones".

La innovación pública tiene como resultado políticas públicas más participativas y transparentes. Si bien todas las políticas públicas son producto de la relación entre diferentes actores y el gobierno, la interrelación que se establece entre ellos, y las instituciones que configuran su gobernanza no son siempre visibles (Tsebellis 2006); en ese sentido, el enfoque central de la innovación pública es ampliar la visibilidad de los actores, su interacción y las instituciones, permitiendo la participación de nuevos actores, ampliando las redes y promoviendo la formación de la

8 La gobernanza es un concepto que sigue siendo discutido en torno a su definición. Entrar en la discusión excede los objetivos planteados por este trabajo, por lo que la entenderemos como el conjunto de reglas y normas que permite y organiza la cooperación y la acción colectiva para la resolución de problemas o conflictos comunes, en un contexto sin límites claros entre empresas, gobierno y sociedad civil, por lo que la comunicación entre actores es más horizontal, sin que un actor se imponga por encima de los demás al reconocer que la cooperación necesita la participación de todos (Ostrom 2000: 99, Swyngedouw 2005: 1995, González y Healey 2005: 2052, Mothe 2006: 97). Entendemos por gobernanza de la red a la configuración del clúster, es decir el conjunto de actores que participa en la red y que comparten valores, creencias y cultura alrededor del paradigma; es decir, la normalización en la relación entre actores que conduce al equilibrio (Börzel, 1998, Marsh y Smith 2000). 
acción pública9 a través de la suma de esfuerzos entre sociedad y gobierno.

La diferencia entre los conceptos de innovación gubernamental e innovación pública busca enfatizar, en el caso de la segunda, el establecimiento de nuevas relaciones entre el gobierno y otros actores sociales. El concepto de innovación gubernamental, al igual que el concepto de innovación, reconoce al gobierno como una organización inserta en un contexto en dónde se establecen relaciones con diferentes actores, por lo que el concepto de innovación gubernamental abre la puerta a reconocer las relaciones entre gobierno y sociedad a través de políticas públicas innovadoras; sin embargo, el concepto de innovación pública enfatiza el papel de la gestión pública participativa como elemento central (Marińez 2015: 13).

Lainnovación pública implicay trasciendealainnovación gubernamental. A partir del reconocimiento de que los gobiernos se encuentran en una compleja red organizacional y que la innovación implica la transformación organizacional, en contextos democráticos la innovación pública conduce a nuevas relaciones entre sociedad y gobierno. En contextos donde la sociedad es más exigente, la participación promueve nuevos esquemas para la colaboración en todas las etapas del ciclo de políticas públicas, para que los gobiernos implementen políticas con un mayor impacto en la calidad de vida de su población, con un mejor y más transparente uso de los recursos públicos (Mariñez 2015).

La interacción de actores con el gobierno, tiene como producto la construcción de paradigmas de política pública, entendidos como la forma como se definen los problemas, las soluciones disponibles, las políticas

9 Entendemos por acción pública "la construcción y la calificación de los problemas colectivos por una sociedad, problemas que ella puede delegar o no delegar a una o varias agencias gubernamentales, en su totalidad o en parte, así como la elaboración de respuestas, contenidos, instrumentos y procesos para su tratamiento" (Thoenig, citado por Cabrero 2005: 19). 
que se implementan, los instrumentos empleados ${ }^{10} \mathrm{y}$ su red de actores ${ }^{11}$ (Sabatier y Jenkins-Smith; 2003); en otras palabras, la definición de los paradigmas de las políticas públicas, es producto del conflicto de los actores que participan en la red y de su capacidad para emplear sus recursos y formar las coaliciones que permitan la construcción y legitimación del paradigma $^{12}$ (Cabrero 2003, Sabatier 2007); en ese sentido, a partir de reconocer que la innovación implica la ruptura y construcción de paradigmas, la innovación pública se observa a través de la constitución de nuevas coaliciones, de manera más transparente y con una amplia participación.

La interacción de actores en la red de la política implica la confrontación/ negociación de sus intereses y recursos disponibles. El equilibrio en las redes depende de los recursos de cada actor y su capacidad para formar coaliciones, destacando entre ellos el nivel de conocimiento respecto de la materia (Baumgartner y Jones 1991, Hirschi y Widmer 2010), aquellos que cuenten con la capacidad de influir en la definición y legitimidad del paradigma de la política se constituyen como actores con poder de veto (Howlett y Ramesh 2003, Tsebellis 2006), por lo que a mayor especialización requerida en un determinado asunto, menor será el número de actores con poder de veto y tenderá a configurar monopolios o triángulos de hierro; pero en asuntos que requieran menor especialización habrá un mayor número de actores, configurando redes y comunidades

10 Entendemos por instrumentos de política al conjunto de "[...] técnicas de las que dispone el gobierno para implementar sus políticas públicas y lograr sus objetivos" (Howlett 1995: 53). El uso de los instrumentos implica la relación entre el gobierno, que brinda un producto o servicio, con la sociedad que lo demanda y evalúa.

11 La red de la política pública se entiende como el conjunto de interacciones entre actores que definen y legitiman el paradigma de la política (de la Mothe 2002: 257). Las redes se constituyen como clústers de actores individuales o colectivos, a través de vínculos de dependencia hacia los recursos con que cuente cada actor, enfatizando la importancia de los actores y los vínculos entre ellos (Carlsson 2000). Al hacer referencia a los vínculos entre actores, establecidos por el intercambio de recursos, configuran las redes en torno a valores, creencias y una cultura compartida alrededor del paradigma de la política; los cambios en la red, se harán a partir de cambios en los valores de los actores (Börzel 1998, Marsh y Smith 2000). A partir del número de actores con poder de veto sobre el paradigma se configuran triángulos de hierro/ monopolios, redes o comunidades de políticas (Cabrero 2003).

12 Los actores se relacionan entre sí por materias de interés, no por secretarías o ministerios, ni por niveles u órdenes de gobierno, por lo que los actores trascienden estos límites (Cabrero 2003, Howlett 2009, Howlett y Ramesh 2003, Carter 2000). 
amplias (Cabrero 2003, Sabatier 2007, Howlett y Ramesh 2003).

Los cambios en el contexto (eventos inesperados, desarrollos tecnológicos, nuevos actores) obligan constantemente a ajustar el paradigma y mantener el equilibrio. Para ello, reconociendo la limitación en los recursos gubernamentales, los cambios pueden derivar en ajustes marginales en los costos asumidos por los actores (incrementales) o en la construcción de nuevas instituciones, completamente diferentes. La diferencia entre los cambios incrementales y los abruptos, depende de la capacidad de los primeros para brindar los resultados esperados; cuando dichos ajustes no tienen la capacidad de alcanzar el bien, resulta evidente la necesidad de nuevas instituciones que logren satisfacer dicha necesidad. Para explicar el proceso de cambio en la red de actores, seguiremos el modelo propuesto por Carter (2000), el cual consta de las siguientes etapas: 1) estabilidad del régimen, el cual implica la vigencia de un paradigma estable, legítimo, aceptado por los actores en la red; 2 ) el surgimiento de detonadores (triggers, enablers) que alteran los incentivos de los actores, los cuales pueden ser consecuencia de cambios en los recursos de los actores o elementos del contexto; 3) fragmentación de la autoridad, cuando el equilibrio de actores es cuestionado por nuevos actores, cuestionando también el paradigma vigente; 4) crisis de legitimidad, cuando la coalición de actores intenta ajustarse (incorporando nuevos actores a la coalición dominante) y ajustar marginalmente el paradigma frente a las críticas (Baumgartner y Jones 1991). En esta etapa, los procesos político-electorales pueden ser aprovechados por los promotores de nuevas coaliciones para tratar de redefinir el paradigma (Sabatier 2007). Las coaliciones vigentes pueden tener éxito en los ajustes marginales que hubieran impulsado, legitimar nuevamente el paradigma y concluir el proceso con un nuevo equilibrio; 5) cambios en la integración de la coalición promotora del paradigma de la política, consecuencia de la crisis de legitimidad; y 6) institucionalización de un nuevo modelo de gobernanza, es decir, implantación y adopción de nuevas reglas y valores en la red de la política.

A partir de la exposición realizada en este apartado, recordando que el objetivo de la investigación es el análisis de la política de seguridad en Nuevo León en el periodo 2009-2015, se toman las consideraciones siguientes. En primer lugar, asumimos como innovación un producto organizacional que implica la ruptura entre paradigmas. En segundo lugar, reconocemos que la innovación es producto de la cooperación y la suma de esfuerzos. En tercer lugar, señalamos la necesidad de que la innovación sea un proceso sistemático, no excepcional, lo cual requiere de una transformación organizacional que lo permita y de agentes promotores que la impulsen y conduzcan, considerando que si esto no se logra, la innovación tenderá a 
ser obsoleta y a que los recursos invertidos en su desarrollo constituyan un desperdicio. En cuarto lugar, diferenciamos los conceptos de innovación gubernamental y de innovación pública, considerando al primero como una transformación hacia dentro del gobierno y al segundo orientado a la transformación en las relaciones del gobierno con la sociedad. En consecuencia, al abordar como tema específico la innovación pública para la seguridad en Nuevo León, el análisis se enfoca en exponer los cambios en la gobernanza de la red de la política pública de seguridad, específicamente la interacción entre actores.

Para el desarrollo de la investigación se emplearon las siguientes herramientas. Para construir y exponer la evolución de la crisis de seguridad, el paradigma de la política en la materia y los ajustes realizados para enfrentarla, se realizó una investigación documental hemerográfica a través de los diarios locales más importantes, Milenio y El Norte, además de revisar el semanario Proceso en el periodo 2003-2015, el cual abarca los periodos de los gobernadores Natividad González Parás y Rodrigo Medina de la Cruz. La exposición y análisis de las estrategias gubernamentales implicaron reconocer la existencia de una estrategia declarada y una estrategia real (Bailey 2010). Para observar la estrategia declarada, se realizó una revisión de los planes de gobierno y programas sectoriales específicos. Para observar la estrategia real, se realizó un seguimiento de los discursos de los gobernadores, a través de la revisión hemerográfica mencionada, así como de las versiones estenográficas de los mismos y boletines de prensa girados por las oficinas gubernamentales. Adicionalmente, con la finalidad de documentar elementos de la estrategia que carecen de una demostración documental, durante el periodo de agosto-diciembre del 2014 se realizaron entrevistas semiestructuradas al comisario de la Policía Estatal, Felipe de Jesús Gallo; al asesor de seguridad que el Gobierno Federal envió al estado de Nuevo León, Jorge Tello Peón; y a la presidenta de la organización Ciudadanos en Apoyo a los Derechos Humanos (CADHAC), Consuelo Morales; entrevistando a cada uno de ellos dos veces para corroborar la información. Finalmente, para construir el epílogo de este trabajo, en el mes de septiembre del 2015 se entrevistó al gobernador Jaime Rodríguez Calderón y al coordinador del Poder Ejecutivo Fernando Elizondo.

El elemento central para el análisis es la innovación pública reflejada en la transformación de las relaciones entre sociedad y gobierno alrededor de la política de seguridad. Para ello, a continuación se expondrá la crisis de seguridad en Nuevo León y las herramientas empleadas para enfrentarla, destacando la construcción de una nueva policía estatal, a través de la suma de esfuerzos entre el sector empresarial, instituciones educativas y el gobierno del estado; así como una nueva forma de atender a las 
víctimas de la delincuencia, especialmente relacionadas con desapariciones forzadas, a través de la colaboración entre el gobierno y organizaciones no gubernamentales defensoras de los derechos humanos.

\section{INNOVACIÓN PÚBLICA PARA LA SEGURIDAD DEL ESTADO DE NUEVO LEÓN}

La región noreste de México, integrada por los estados de Coahuila, Nuevo León, San Luis Potosí y Tamaulipas no ha ocupado un espacio relevante en la historia del crimen organizado en México. El estado de Tamaulipas es el único de la región que tradicionalmente ha tenido un papel relevante para las organizaciones criminales, debido a las fronteras que comparte con los Estados Unidos, lo cual también ha convertido al estado un escenario conflictivo y violento ante los ajustes de las organizaciones criminales (Astorga 2015, Valdés 2013); el resto de los estados no habían sido partícipes de esta guerra y solo habían resentido impactos marginales. Sin embargo, en el periodo de los ańos 2009-2015, en respuesta a cambios en la política contra las drogas en Estados Unidos, así como por el enfrentamiento del gobierno mexicano contra las organizaciones criminales (Montero 2010, Bailey 2014, Calderón 2014), la región enfrentaría la peor crisis de seguridad en su historia contemporánea, destacando el caso de Nuevo León que enfrentó el mayor incremento en el número de homicidios dolosos en la región, pero que también fue el que proporcionalmente redujo dicho número, tal como se puede observar en el siguiente gráfico.

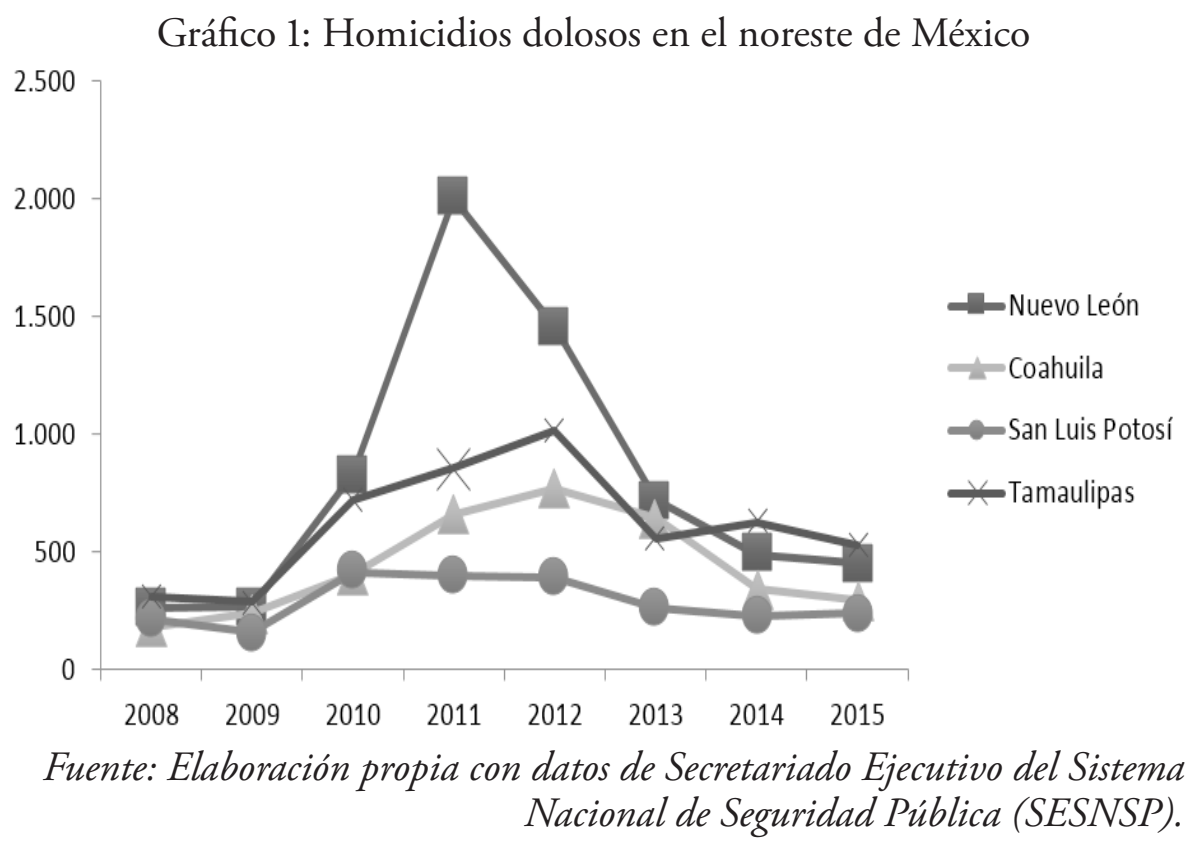


El comportamiento de los homicidios dolosos en Nuevo León es lo que ha llevado a la presente investigación al estudio de su política pública en materia de seguridad, llegando a la identificación de diferentes elementos, pero destacando dos de ellos el desarrollo de la nueva policía estatal y un mecanismo innovador para la atención a víctimas de la delincuencia, especialmente relacionadas con desapariciones forzadas; ambos casos, construidos a partir de la cooperación entre sociedad y gobierno, constituyendo ejemplos de innovación pública en una materia que tradicionalmente se considera como un monopolio del gobierno, mismos que expondremos a continuación.

El estado de Nuevo León no había sido un espacio importante para el crimen organizado. Ha sido señalado como centro financiero de organizaciones criminales o donde pueden residir los líderes de diferentes organizaciones sin agredirse mutuamente, tal como lo han demostrado en sus investigaciones Astorga (2007 y 2015), Valdés (2013), Ravelo y Salas (2006) y Campos (2012), entre otros; en consecuencia, ha sido un espacio pacífico, únicamente alterado por eventos violentos poco frecuentes asociados a conflictos entre organizaciones criminales que tienen su mayor expresión en el vecino estado de Tamaulipas, debido a que en dicho estado se encuentran las tres principales fronteras del noreste de México con los Estados Unidos. En la misma dirección, la lucha entre organizaciones criminales, así como las acciones del Gobierno Federal en contra de las organizaciones criminales, impactan de manera directa e indirecta en el estado de Nuevo León.

La seguridad en el estado ha enfrentado dos crisis importantes. La primera, durante el gobierno de Natividad González Parás, entre los años 2005-2007; y la más severa, durante el inicio del gobierno de Rodrigo Medina de la Cruz, entre los ańos 2009-2011, tal como se presenta en el siguiente gráfico. 
Gráfico 2: Número de homicidios en Nuevo León

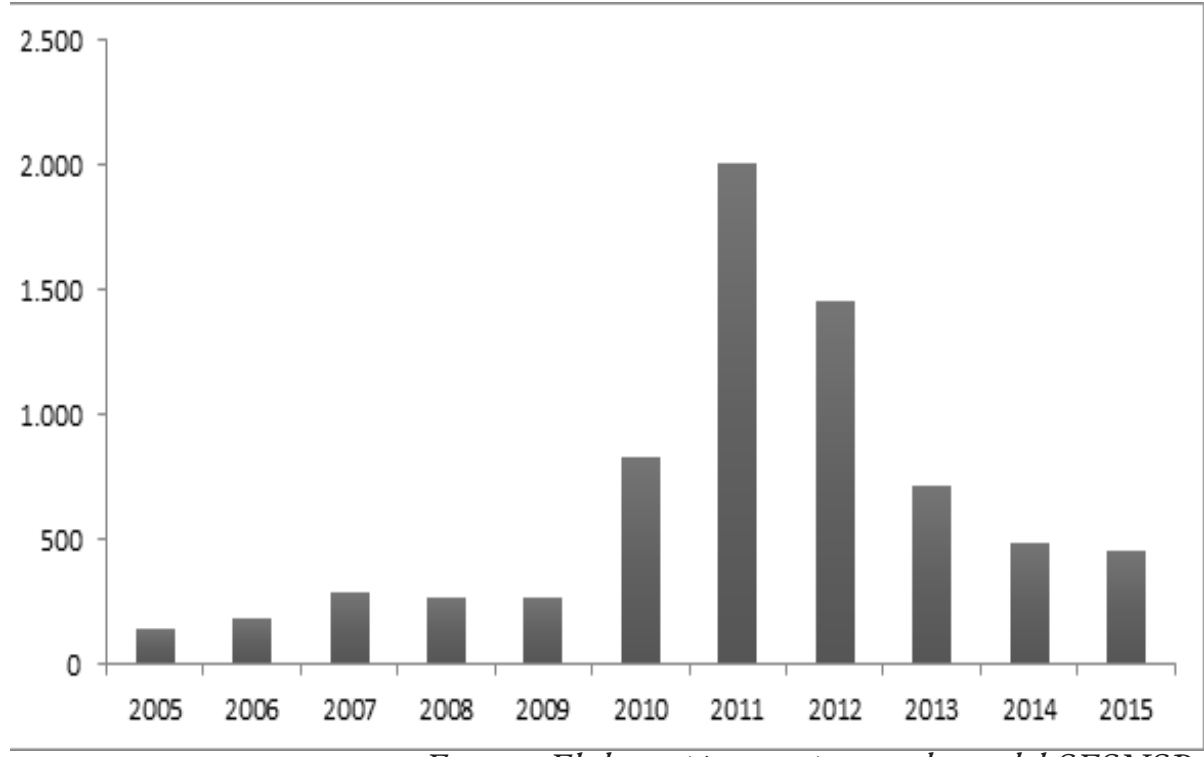

Fuente: Elaboración propia con datos del SESNSP.

La seguridad en México constituye una materia compleja, que requiere un alto nivel de conocimiento y especialización, por lo que la red de actores alrededor de la política pública constituye un monopolio controlado principalmente por los responsables de la seguridad interior (secretaría de gobernación), policías, inteligencia y fuerzas armadas (Montero 2013). Al ser la política manejada por un monopolio, los cambios y ajustes en la política de seguridad en Nuevo León se realizaban de forma marginal, especialmente ante la presión de la prensa, sin cambios abruptos. Sin embargo, el incremento de la violencia, especialmente cuando afectó directamente a familias de empresarios, constituyó el disparador de los cambios en el paradigma al incorporar a los empresarios como actores que tradicionalmente no se involucraban en la materia, pero que a partir de verse afectados por la inseguridad, participaron en la constitución de movimientos sociales para presionar al gobierno y exigir mejores resultados en el diseño e implementación de las estrategias de seguridad.

Durante la primera crisis, la estrategia del gobernador González Parás (2003-2009) consistió en: 1) expresar públicamente que la violencia no se realizaba en Nuevo León sino en Tamaulipas, por lo que no era problema de Nuevo León; 2) expresar también que las la violencia que sí se registraban en el estado, podía enfrentarse, sin necesitar el apoyo del Gobierno Federal, entonces encabezado por el presidente de la República, Vicente Fox (2000-2006); 3) declarar que la atención del problema correspondía al Gobierno Federal, a pesar de que pudiera ser contradictorio con el 
inciso anterior; y 4) con la finalidad de tratar de incorporar a la ciudadanía en la red de la política pública de seguridad, se constituyó un consejo ciudadano de seguridad pública, pero que sin facultades vinculantes para el gobierno e integrado para favorecer al gobernador del estado, por lo que no era una participación real sino controlada por el gobierno (Fernández 2009, Villarreal 2009). En resumen, el gobierno minimizaba el problema, rechazaba solicitar apoyo del Gobierno Federal, pero al mismo tiempo lo responsabilizaba de enfrentar a las organizaciones criminales, expresaba que su gobierno tenía la capacidad de enfrentar la parte del problema que le correspondería y cooptaba a la ciudadanía a través del consejo ciudadano.

A pesar de los cuestionamientos por la falta de resultados, el gobierno mantuvo su postura e impulsó ajustes marginales para legitimar nuevamente su paradigma. Los ajustes no lograron los resultados esperados y se tuvieron que impulsar cambios más radicales, pero el cambio en la estrategia no provino del gobierno del estado, lo impulsó el Gobierno Federal. Con la llegada del presidente Felipe Calderón (2006-2012), el Gobierno Federal envió a Nuevo León a un importante número de militares, policías federales, ministerios públicos, peritos, policías ministeriales y asesores ${ }^{13}$.

Durante el 2009, la crisis de seguridad de Nuevo León coincidió con el proceso electoral local para la renovación de alcaldías, congreso local y la gubernatura del estado. Inicialmente, el nuevo gobierno del estado encabezado por Rodrigo Medina de la Cruz (2009-2015), mantuvo la estrategia de responsabilizar al Gobierno Federal de la lucha contra las organizaciones criminales. Sin embargo, entre 2009 y 2011 explotó la crisis: el número de homicidios creció más de $700 \%$, además de la ocurrencia de balaceras en la ciudad de Monterrey a plena luz del día, donde los narcotraficantes demostraron su capacidad para enfrentar a la policía federal y al ejército; se incrementaron los asesinatos y desapariciones forzadas de policías municipales en la Zona Metropolitana de Monterrey y municipios rurales del estado (Buendía, Charles y García 2010); se expuso la participación de policías municipales en apoyo a los criminales contra el ejército; se volvió frecuente el asesinato de personas inocentes entre quienes destacan estudiantes del Tecnológico de Monterrey y de la Universidad Autónoma de Nuevo León; ocurrió el secuestro y asesinato del presidente del municipio de Santiago, Edelmiro Cavazos, entre muchos

13 La colaboración con el Gobierno Federal se puede resumir en la intervención directa, a la que se ha hecho referencia, pero también en inversión en materia de infraestructura tecnológica para la seguridad y coordinación intergubernamental liderada por la federación (Montero 2010, Calderón 2014, Astorga 2015). 
otros eventos ${ }^{14}$.

Adicionalmente, entre el 2009 y 2014 comenzó un fenómeno nunca antes visto: las desapariciones forzadas, registrándose 1.249 personas desparecidas en ese periodo, siendo señaladas las autoridades gubernamentales como responsables de la gran mayoría de estas (CADHAC 2014).

La estrategia del Gobierno Federal contribuyó a incrementar la violencia en el país y en Nuevo León. Lamentablemente, a partir de la intervención de las fuerzas federales, se incrementaron las denuncias por violaciones a los derechos humanos cometidas por las policías municipales, estatales y fuerzas federales, destacando las relacionadas con detenciones injustificadas, tortura, ejecuciones extrajudiciales y desapariciones forzadas; en consecuencia, se incrementaron también los asuntos atendidos por organizaciones defensoras de derechos humanos, así como las recomendaciones emitidas por las Comisiones Estatal y Nacional de los Derechos Humanos, llegando a su punto más álgido en el año $2012^{15}$. En la misma dirección, el incremento en las recomendaciones alimentó la idea de que las organizaciones defensoras de los derechos humanos se convertían en aliados de los criminales y enemigos del gobierno (Monsiváis, Torres y Talavera 2014).

El contexto era muy complejo: por un lado, una guerra entre organizaciones criminales y, por otro, el gobierno enfrentando a las mismas organizaciones. La ciudadanía quedó en medio de estalucha, dondelos daños colaterales y las violaciones a derechos humanos comenzaron a convertirse

14 Debe hacerse un importante apunte sobre la sociedad civil en Nuevo León. A diferencia de otras expresiones a nivel nacional, en Nuevo León, el papel de los empresarios es determinante debido a su influencia. En el estado se asientan los corporativos de tres de las empresas más importantes de México: Cementos Mexicanos (CEMEX), Fomento Económico Mexicano (FEMSA) y Grupo Alfa, pero también otro conjunto muy importante de empresas que convierten al gremio empresarial en uno de los más importantes del país, con influencia local, regional y nacional.

$15 \mathrm{Al}$ respecto, destacan las recomendaciones de la Comisión Estatal de Derechos Humanos número 013 y 015 del 2011, 50, 51, 52, 55 y 56 del 2013, las cuales abordan casos de desaparición forzada, así como las número 13 y 15 del 2011, relacionadas con ejecuciones extrajudiciales; en el mismo sentido, se realizaron las recomendaciones número 45/2010, 34/2011, 55/2012 y 43/2013 de la Comisión Nacional de los Derechos Humanos, todas relacionadas con desapariciones forzadas. De la misma manera, en el periodo del 2009-2014, que abarca la crisis de seguridad, el número de casos atendidos por CADHAC se incrementaron a un ritmo de 7\% anual, destacando los casos de desaparición forzada y ejecuciones extrajudiciales. 
en un elemento importante. Debido a que tradicionalmente existe una desvinculación entre la ciudadanía y el gobierno por ser la red de seguridad un monopolio, la forma como la ciudadanía expresó su descontento y cuestionó la estrategia, fue a través de la construcción de movimientos sociales y organizaciones no gubernamentales. Los movimientos sociales que surgieron frente a la crisis de seguridad pueden clasificarse en tres grandes grupos: el primero, impulsado por las propias organizaciones criminales, usando a la sociedad civil para protestar contra la presencia del Ejército Mexicano y empleando como argumento las violaciones a los derechos humanos por parte de los militares ${ }^{16}$; en segundo lugar, aquellos liderados por empresarios que fueron víctimas de la delincuencia, menos visibles pero muy influyentes; y en tercer lugar, los liderados por víctimas de la delincuencia, organizaciones defensoras y promotoras de los derechos humanos, más numerosos, pero menos influyentes. La diferencia entre los dos últimos, es la disponibilidad de recursos con que cuentan los empresarios (económicos e influencia política) para constituirse como actores con poder de veto, pero que por su cercanía con el gobierno les ha llevado a ser señalados como activistas legitimadores de la estrategia gubernamental (Steels 2015); los últimos, son más críticos, no son cercanos al gobierno y la interacción entre ambos es excepcional, generalmente consecuencia de la presión a través de manifestaciones (Monsiváis, Torres y Talavera 2014).

Ante la crisis de seguridad y la presión social, el gobierno del estado se vio obligado a reconocer la magnitud del problema y cambiar su estrategia. En primer lugar, impulsó la creación de un sistema de inteligencia, la capacitación de los policías ministeriales y modernización de las áreas técnicas para la investigación de los delitos, la creación de una nueva unidad anti-secuestro y la transformación de la academia de policía en la Universidad de Ciencias de la Seguridad, entre las más importantes; adicionalmente, con apoyo del Gobierno Federal, se fortalecióla certificación de todos los cuerpos de policía; sin embargo, estas transformaciones no implicaron una nueva forma de relacionarse entre sociedad y gobierno, por lo que deben considerarse casos de innovación gubernamental y no de innovación pública, por lo que serán dejadas de lado, sin dejar de tomarlas en cuenta como parte de la transformación de la política en materia de

16 Entre el 8 y 11 de febrero del 2019 se realizaron manifestaciones encabezadas por personas con el rostro cubierto, denunciando presuntas violaciones a los derechos humanos cometidas por las fuerzas federales y exigiendo su retiro del estado; en respuesta, los gobiernos estatal y federal denunciaron que los organizadores de las manifestaciones eran los propios delincuentes. Si bien no constituyeron un movimiento social, constituyeron un fenómeno excepcional a considerar en el caso. 
seguridad. En segundo lugar, ante la presión social, implementó dos estrategias. La primera, respecto de los empresarios y las universidades, las incorporó en la Alianza por la Seguridad, para que participaran en el diseño y evaluación de la política pública; la segunda, respecto de los movimientos de víctimas de la delincuencia y defensores de los derechos humanos, el gobierno inicialmente los ignoró e incluso los consideró como adversarios políticos por las críticas que recibía; sin embargo, después de la visita del Movimiento por la paz, la justicia y dignidad en junio del 2011, ante una manifestación frente a la Procuraduría General de Justicia, donde se expusieron y denunciaron varios casos de desaparición forzada, el procurador accedió a dialogar con los líderes del movimiento y a comenzar una relación de diálogo entre la autoridad y la organización CADHAC para analizar de manera conjunta los avances en materia de búsqueda de las personas desaparecidas.

Ambas estrategias configuran casos de innovación pública debido al nivel de involucramiento de los actores sociales que transformó la red de la política de seguridad. Entre los elementos diferenciadores de esta colaboración, destaca que los empresarios se involucraron en la implementación y el financiamiento de la política. Por otro lado, la relación entre el Gobierno Federal y los movimientos de víctimas logró hacerse cotidiano e incluso ha trascendido el periodo gubernamental, las organizaciones sociales hicieron suya esta nueva forma de trabajar con el gobierno y no han permitido que se abandone.

La colaboración entre el movimiento de víctimas y el gobierno del estado ha tenido como productos, en primer lugar la sistematización de la relación entre la Procuraduría y las familias de las víctimas del delito de desaparición forzada para la revisión de expedientes; la tipificación de delito de desaparición forzada que permitió crear la figura legal de desaparecido y que implicó la colaboración entre organizaciones sociales y el Congreso del Estado de Nuevo León ${ }^{17}$; la creación de protocolos para el trámite y atención de denuncias para la búsqueda inmediata de personas desaparecidas, la cual a su vez generó el desarrollo de un grupo especial denominado Grupo Especializado de Búsqueda Inmediata (GEBI), el cual ha permitido al estado responder positivamente a este tipo de denuncias (CADHAC 2014). Todos estos productos, han sido consecuencia de un modelo de colaboración entre sociedad y gobierno, superando la consideración que tenía cada actor respecto del otro como adversarios y

17 Esta reforma legal se promulgó en el 2012, un año antes de que se promulgara la Ley de Víctimas a nivel nacional, lo cual fortalece el valor de este tipo de colaboración entre sociedad y poder legislativo en Nuevo León. 
sumando esfuerzos para atender el problema, configurando un caso muy claro de innovación pública.

El producto que ha tenido mayor trascendencia ha sido el desarrollo de la nueva policía estatal Fuerza Civil, producto de la triple hélice formada entre empresas, universidades y gobierno, a través de la conformación de la Alianza por la Seguridad como mecanismo de colaboración entre grupos empresariales, las principales universidades del estado y el gobierno, por lo que es necesario exponerla con mayor detalle.

A inicios del 2011 se constituyó formalmente la Alianza por la Seguridad, con el objetivo de contribuir en el reclutamiento de policías estatales. La gran diferencia de la Alianza por la Seguridad, frente a cualquier otro caso de cooperación en México, es que los empresarios de Nuevo León se comprometieron a brindar recursos al estado para la realización de un diagnóstico ${ }^{18}$ del problema, la elaboración de propuestas, el reclutamiento del personal, la certificación policial, el equipamiento, el desarrollo de infraestructura y la evaluación al desempeño gubernamental (Salazar 2013). Entre los resultados del diagnóstico se consideró que debido a la desconfianza de la ciudadanía hacia la policía, resultaba necesario crear una nueva institución, con un nuevo concepto, incluso con otro nombre. En consecuencia, se decidió crear la Fuerza Civil, acompañada con una importante campaña de marketing y comunicación que destacaría la profesionalización y certificación de sus integrantes, separándola con claridad de otras policías, pero cuidando de no violentar los criterios establecidos por el Sistema Nacional de Seguridad Pública y por el Sistema Integral de Desarrollo Policial.

La Alianza por la Seguridad incorporó a empresarios, representantes de las universidades y de la sociedad civil (especialmente de asociaciones encabezadas por empresarios), con el gobierno del estado. Ante el clima de crispación, la Alianza constituyó un medio para canalizar las

18 El nombre Fuerza Civil es consecuencia de dicho diagnóstico y del problema enfrentado en el reclutamiento de policías. Las empresas incorporadas en la Alianza se involucraron en el proceso de reclutamiento con escasos resultados, debido a que ser policía estatal seguía siendo poco atractivo y los requisitos eran muy altos. Para enfrentar el problema, los empresarios se comprometieron a invertir para hacer más atractiva la labor del policía estatal, ofreciendo uno de los salarios más atractivos del país, vivienda, prestaciones excepcionales; para crear una nueva marca para la policía con el nombre de Fuerza Civil, el cual a través de las estrategias de marketing buscaría separarse completamente de la idea de las policías tradicionales; y para llevar el reclutamiento a diferentes estados de la República (Salazar 2013). 
demandas sociales directamente hacia el gobierno, lo cual contribuyó a reducir las críticas políticas hacia el tema de seguridad, generando una imagen de unidad entre sociedad y gobierno. Además, la Alianza no se limitaría a ser un espacio de diálogo entre la sociedad y el gobierno sino que permitió la construcción de acuerdos y compromisos mutuos para el diseño e implementación de nuevas estrategias en materia de seguridad. La suma de esfuerzos entre sociedad y gobierno, incorporando actores que tradicionalmente no pertenecen a la red de la política de seguridad (universidades, ciudadanos y empresarios), transformaron las interacción alrededor de la política, configurando el caso de innovación pública; sin embargo, carecen de uno de los elementos característicos de este tipo de innovación: los mecanismos de transparencia, evaluación y rendición de cuentas $^{19}$.

El nuevo modelo de policía estatal se hizo a semejanza del modelo de la policía federal implementado durante la gestión del presidente Felipe Calderón, en el cual se destaca no solo la necesidad de la capacitación constante y la modernización de la infraestructura sino la incorporación de labores de inteligencia para la actuación policial (García 2011). En consecuencia, Fuerza Civil incorporó no solo personal operativo sino también análisis e investigación, es decir, inteligencia, tal como lo expone el Gobierno del Estado de Nuevo León (2012), en su tercer informe de gobierno. Otro elemento diferenciador es la capacitación que recibe el personal operativo, quienes son adiestrados y capacitados por la Secretaría de la Defensa Nacional en cuarteles militares, por la Academia Superior de Seguridad Pública de la Secretaría de Seguridad Pública Federal y finalmente en la Universidad de Ciencias de la Seguridad (anteriormente Academia de Policía). De manera complementaria, el gobierno del estado realizó inversiones en la adquisición de infraestructura y recursos necesarios para la labor policial como uniformes especiales, vehículos, armamento y sistemas de información, entre los más importantes. Entre los principales objetivos de Fuerza Civil se encuentran: 1) la capacidad de reacción ante emergencias ocasionadas por grupos de la delincuencia; 2) combatir el delito apoyados de inteligencia policial; y 3) generar confianza entre la población a partir del profesionalismo en su actuación y con el rigor en su reclutamiento.

19 Las reuniones de la Alianza por la Seguridad carecen de minutas o documentos públicos en donde se transcriban los debates o acuerdos; tampoco existen compromisos públicos, ni reportes de los recursos ejercidos por los involucrados. En ese sentido, para la construcción del caso, nos hemos apoyado de la revisión hemerográfica, así como de las entrevistas ya referidas. 
La Alianza por la Seguridad, que derivó en el desarrollo de Fuerza Civil, creó una institución legítima que no ha sido criticada por ninguna fuerza política, alejándola también de discusiones políticas y el propio uso discrecional por parte del gobierno del estado debido a que Fuerza Civil no puede atribuírsele al gobierno del estado sino a la suma de esfuerzos públicos. Una vez en funciones, diversos hechos pusieron a prueba a la nueva policía estatal. El primer caso fue el incendio del Casino Royale, que tuvo como consecuencia 52 personas muertas, pero donde Fuerza Civil lograría aprehender rápidamente a los autores materiales e intelectuales del incendio. Una segunda prueba fue la masacre de 44 presos y fuga de 30 más del penal de Apodaca, donde Fuerza Civil se haría responsable de enfrentar la crisis de una manera incuestionable, sin críticas por parte de grupos políticos o sociales ${ }^{20}$. Para el 2013, Fuerza Civil se había convertido en uno de los proyectos más importantes y exitosos del gobierno de Rodrigo Medina, lo cual se expresa con el nivel de confianza de la sociedad hacia la policía, tal como se expresa a continuación.

20 Debe separarse la opinión hacia la actuación de Fuerza Civil y otra la vertida hacia la administración del penal. A pesar de que ambas funciones corresponden a la Secretaría de Seguridad Pública, nuestro enfoque es hacia Fuerza Civil. La administración de los penales ha sido fuertemente criticada y lamentablemente no se ha hecho mucho para corregirlo, una gran parte de las recomendaciones de la CEDH se dirige a estas instituciones y, de manera específica, respecto al motín en el penal de Apodaca, la CNDH emitió la recomendación 40/2013. 


\section{Gráfico 3: Confianza en la Policía Estatal}

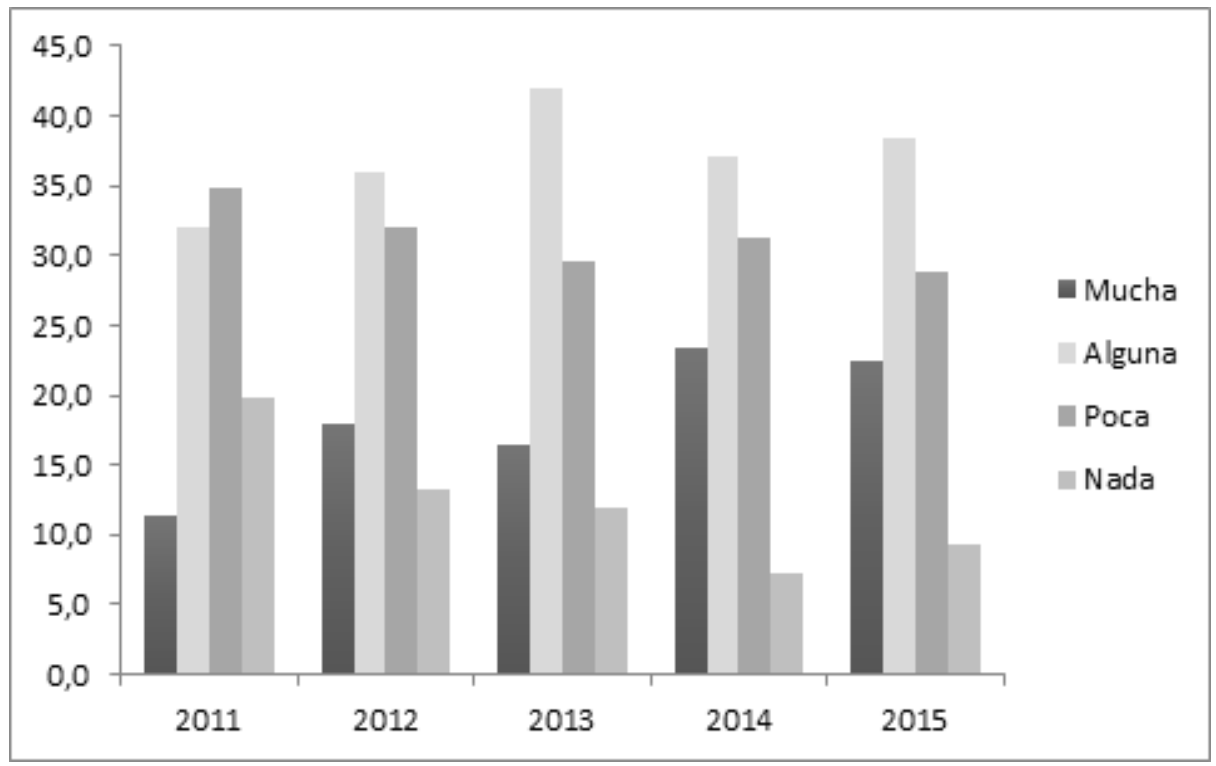

Fuente: Elaboración propia con datos de la Encuesta Nacional Sobre Victimización y Percepción de la Seguridad ${ }^{21}$.

En la misma dirección, los presidentes Felipe Calderón y Enrique Peña han reconocido a la policía estatal, señalándola como modelo a seguir por el resto de los estados ${ }^{22}$. Sin embargo, la nueva policía estatal enfrenta retos importantes. En primer lugar, destaca el financiamiento, el cual depende de acuerdos informales, no institucionalizados, entre el gobierno del estado y los empresarios. En segundo lugar, después del proceso electoral del 2015, el gobernador Jaime Rodríguez Calderón (2015-2021) no ha convocado nuevamente a la Alianza, lo cual puede conducir a su desaparición y que se pierdan los apoyos brindados por los empresarios. En tercer lugar, el actual gobernador no ha expuesto sus intenciones con Fuerza Civil, e incluso se ha incrementado la presencia militar en el estado, lo cual permite presumir que el gobierno no invertirá más en la policía estatal. En cuarto lugar, es necesario el fortalecimiento de las policías municipales o el desarrollo de unidades de policía de proximidad, toda vez que Fuerza Civil está diseñada para realizar labores de reacción inmediata, choque, pero no de prevención.

21 La encuesta se comenzó a realizar en el 2011, por lo que se carece de datos previos.

22 A partir de estos señalamientos, prácticamente todos los gobernadores han acudido a conocer el proyecto de Fuerza Civil y se ha tratado de replicar en estados como Coahuila, Tamaulipas, Veracruz, entre otros. 
La Alianza por la Seguridad, que tuvo como resultado a la nueva policía estatal, es un caso exitoso de innovación pública. Sin embargo, carece de dos elementos importantes: la sistematización de la colaboración que permita que la innovación no sea un producto aislado, extraordinario sino constante; y los mecanismos participativos para evaluar la política, garantizar la transparencia y la rendición de cuentas. No se crearon organismos responsables de asegurar la conservación de la alianza, lo que ha permitido que ante el cambio de gobierno la interacción y cooperación entre actores se diluya y desaparezca, lo cual rompe significativamente con las características de la innovación pública. Asimismo, la falta de este tipo de mecanismos, reduce las posibilidades de que el cambio organizacional se implante, abriendo la posibilidad de que se revierta y, al no hacer de la innovación un proceso sistemático, que la nueva policía estatal quede obsoleta rápidamente. Tampoco se crearon mecanismos ciudadanos para monitorear y evaluar la política de seguridad y retroalimentar la política, por lo que se carece de herramientas para identificar y corregir los problemas, abriendo la puerta para que la policía caiga en viejos vicios de las policías en México, sin contar con la red de actores que permitieran emplear la innovación pública como mecanismo para corregirlos.

La principal lección que puede dejar el caso de Nuevo León para otros estados que están desarrollando policías estatales de manera similar, es la necesidad de transformar la red de la política de seguridad y generar acuerdos entre sociedad, gobierno y empresarios, lo cual caracteriza a la innovación pública. Sin embargo, dichos acuerdos deben involucrar activamente a la sociedad y a los empresarios en el desarrollo de las instituciones policiales, rompiendo la idea tradicional de que es una responsabilidad exclusiva del gobierno, conduciendo a la apropiación de la nueva institución policial por parte de la ciudadanía en general, evitando que las nuevas policías sean vistas como productos ajenos a la ciudadanía, en cambio, la apropiación de la institución policial brindará mayor legitimidad y solidez a la policía estatal.

\section{EPÍLOGO: LOS RETOS PARA LA INNOVACIÓN PÚBLICA EN LA POLÍTICA DE SEGURIDAD}

La crisis en materia de seguridad impulsó un cambio en la política de seguridad en Nuevo León que implicó procesos de innovación gubernamental e innovación pública. La innovación pública se refleja con claridad en la construcción de la nueva policía estatal, la cual se convirtió en un referente para el resto de entidades federativas en México, destacando la suma de esfuerzos entre universidad, empresarios y gobierno en todo el proceso de la política pública. Sin embargo, una vez pasada la crisis de 
seguridad y perdido el sentido de urgencia o necesidad de la cooperación, dicho esfuerzo ha sido abandonado y la policía ha entrado en una etapa de incertidumbre, lo cual abre la puerta al riesgo señalado en el marco teórico sobre la posibilidad de que la política sea obsoleta rápidamente y los recursos invertidos en su desarrollo pudieran constituir un desperdicio.

Un segundo caso de innovación pública lo constituyó la transformación en las relaciones entre el gobierno y las organizaciones protectoras de los derechos humanos, especialmente las relacionadas con temas de desaparición forzada y ejecuciones extrajudiciales. La colaboración para avanzar en la atención a las víctimas, ha sido un ejemplo a nivel nacional, al superar la lógica de reconocerse mutuamente como enemigos y colaborar, teniendo como resultados nuevas leyes y la creación del GEBI. Por otro lado, a diferencia del caso de Fuerza Civil, donde el catalizador -la crisis de seguridad- parece haberse agotado y ha promovido el abandono de la política, al no haberse resuelto los casos de las víctimas, estas han exigido la conservación de la colaboración con el gobierno, incluso a pesar del poco interés que este ha demostrado por involucrarse en el tema. Este caso debe destacarse, debido a que la sociedad hizo suya la innovación pública y se ha esforzado en mantenerla, logrando su trascendencia y posibilitando el surgimiento de nuevas políticas en el futuro.

La estrategia inicial del gobierno de Nuevo León, al igual que la de muchas entidades federativas, frente al incremento de la violencia asociada al crimen organizado, fue la de rechazar la magnitud del problema y de evadir su responsabilidad, señalando al Gobierno Federal como único responsable de enfrentar al crimen organizado. Sin embargo, una vez que la crisis llegó a su punto más álgido, afectando a empresarios, estudiantes universitarios y la ciudadanía en general, la sociedad se involucró de manera más directa con el gobierno en el diseño e implementación de la política pública.

Dentro del marco teórico se expuso que el reto que enfrentan la innovación pública y la innovación gubernamental, es la capacidad de trascender los periodos de gobierno, adhiriéndose a la definición que la considera a la innovación como un proceso sistemático. En este sentido, debido a que el número de homicidios disminuyó significativamente en el periodo 20132014, el nuevo gobierno, encabezado por Jaime Rodríguez Calderón, dejó de considerar prioritario mantener la alianza universidades-empresariosgobierno, materializada en la Alianza por la Seguridad; en consecuencia, Fuerza Civil ha reducido sus vínculos con la sociedad; adicionalmente, en el periodo 2015-2016, la policía ha enfrentado problemas administrativos, demostrando las dificultades presupuestales para su financiamiento, pero 
también legales y de disciplina, debido a que algunos de sus integrantes se han visto relacionados con violaciones a los derechos humanos (especialmente detenciones arbitrarias y lesiones), así como en la comisión de delitos, lo cual ha contribuido a dañar su legitimidad; finalmente, debe agregarse que a partir del último trimestre del 2015 y durante el 2016, el número de homicidios se ha incrementado nuevamente y se ha reducido la confianza hacia la policía. Por otro lado, en lo que respecta a la atención a víctimas de la delincuencia, tal como mencionó, tampoco ha sido un tema prioritario para el nuevo gobierno e incluso ha sido despreciado por el gobernador; pero en este caso han sido las organizaciones de la sociedad civil quienes han exigido que se mantenga la vinculación con el gobierno, por lo que a pesar de haberse disminuido la colaboración, esta se mantiene y la innovación pública subsiste.

La lección de Nuevo León para otras entidades es que la seguridad no es un asunto solo de policías sino de la sociedad en general, requiriendo para ello el involucramiento de la sociedad civil en el diseño e implementación de las políticas de seguridad pública, enfatizando que su papel no concluye con la incorporación de un asunto en la agenda de gobierno sino que requiere del compromiso para involucrarse en todas las etapas de la política pública, destacando la necesidad de mantener la colaboración para garantizar que la innovación pública se conserve, que las políticas no se hagan obsoletas y se conduzcan con transparencia. En el caso de Nuevo León, la ciudadanía se involucró activamente en el diseño, financiamiento e implementación de la política, pero -sobre todo en el caso de la policía estatal-, no se construyeron los mecanismos para la evaluación y rendición de cuentas, ni para conservar la cooperación sociedad-gobierno. La innovación pública para la seguridad constituye un elemento indispensable para lograr resultados diferentes en la lucha por alcanzar la seguridad en México. 


\section{REFERENCIAS}

Arellano, D. (2004). Gestión Estratégica para El Sector Público: Del Pensamiento Estratégico al Cambio Organizacional. México: Fondo de Cultura Económica.

Astorga, L. (2015). ¿Qué Querían que Hiciera? México: Grijalbo. Sombra. México: Tusquets. . (2007). Seguridad, Traficantes y Militares: el Poder y la Bailey, J. (2010). Policy Changes Facing Mexico. Ponencia presentada en Georgetown University, 26 de marzo, Washington.

(2014). Crimen e Impunidad. Las Trampas de la Seguridad en México. México: Debate.

Baumgartner, F. y Jones, B. (1991). Agenda Dynamics and Policy Subsystems. The Journal of Politics, 53 (4), 1044-1074.

Bloch, C. y Bugge, M. M. (2013). Public Sector Innovation- From Theory to Measurement. Structural Change and Economic Dynamics, 27, 133145.

Börzel, T. (1998). Organizing Babylon. On the Different Conceptions of Policy Networks. Public Administration, 76 (2), 253-273.

Buendía, A., Charles, A. y García, J. (2010). Rechaza Ivonne Ligar a Policías Ejecutados con el Narco. El Norte, 18 de Junio.

Ciudadanos Activos por los Derechos Humanos (CADHAC). (2014). Desaparición de Personas en Nuevo León. México: CADHAC-PGJNL.

Campos, L. (2012). De Poder a Poder. En Rodríguez Castañeda, R. (Coord.), El México Narco, México: Planeta.

Cabrero, E. (2003). Políticas Públicas Municipales. Una Agenda en Construcción, México: Miguel Ángel Porrúa-CIDE. de Cultura Económica. 
Cabrero, E., Arellano, D. yAmaya, M. L. (2007). Cambio en Organizaciones Gubernamentales: Innovación y Complejidad. En Arellano, D., Caballero, E. y del Castillo, A. (Cords.), Reformando al Gobierno. Una Visión Organizacional del Cambio Gubernamental, México: CIDE.

Calderón, F. (2014). Los Retos que Enfrentamos. México: Debate.

Carlsson, L. (2000). Policy Networks as Collective Action. Policy Studies Journal, 28 (3), 502-520.

Carter, A. (2000). Policy Regimes and Policy Change. Journal of Public Policy, 20 (3), 247-274.

Carrillo, F. (2015). Knowledge-Based Development as a new Economic Culture. Journal of Open Innovation: Technology, Market and Complexity, $1(15), 1-17$.

Casper, S. y Van Waarden, F. (2005). Innovation and Institutions. Northampton: Edward Elgar Publishing.

Cooke, P., Gomez Uranga, M. y Etxebarria, G. (1997). Regional Innovation Systems: Institutional and Organisational Dimensions. Research Policy, 26 (4-5), 475-491.

Damanpour, F. y Schneider, M. (2009). Characteristics of Innovation and Innovation Adoption in Public Organizations: Assessing the Role of Managers. Journal of Public Administration Research and Theory, 19 (3), 495-522.

De la Mothe, J. (2002). Policy networks in Adaptive Innovation Systems. En de la Mothe J. y Link, A. (Eds.), Networks, Alliances and Partnerships in the Innovation Process. Boston: Kluwer Academic Publishers.

Northhampton: Edward Elgar.

Del Castillo, A. (2007). Problemas en la Acción Gubernamental: Organizaciones y Redes de Actores. En Arellano, D., Caballero, E. y del Castillo, A. (Cords.), Reformando al Gobierno. Una Visión Organizacional del Cambio Gubernamental, México: CIDE.

Downs, A. (1957). An Economic Theory of Political Action in a Democracy. The Journal of Political Economy, 65 (2), 135-150. 
Elder, C. y Cobb, R. (2000). Formación de la Agenda. En Aguilar Villanueva, L. (Ed), Problemas Públicos y Agenda de Gobierno. México: Miguel Ángel Porrúa

Etzkowitz, H. y Leydesdorff, L. (2000). The Dynamics of Innovation: From National Systems and Mode 2 to a Triple Helix of UniversityIndustry-Government Relations. Research Policy, 29 (2), 313-330.

Fernández Garza, H. (2009). Consejo Consultivo Ciudadano de Seguridad Pública de Monterrey. En Mariñez, F. (Coord.), Compromiso Ciudadano. México: Fondo Editorial Nuevo León.

Florida, R. (2011). El Gran Reset: Nuevas Formas de Vivir y Trabajar. Barcelona: Paidós.

García, G. (2011). El nuevo Modelo de Seguridad, México: Rostra Editions.

García R. y Calantone, R. (2002). A Critical Look at Technological Innovation Typology and Innovatiness Terminology: A Literature Review. The Journal of Product Innovation Management, 19 (2), 110-132.

Gerometta, J., Häussermann, H. y Longo, G. (2005). Social Innovation and Civil Society in Urban Governance: Strategies Forming Inclusive City. Urban Studies, 42 (11), 2007-2021.

Girouard, J. y Sirianni, C. (2014). Varieties of Civic Innovation: Deliberative, Collaborative, Network, and Narrative Approaches. Nashville: Vanderbilt University Press.

González, S. y Healey, P. (2005). A Sociological Institutionalist Approach to the Study of Innovation in Governance Capacity. Urban Studies, 42 (11), 2055-2069.

Grady, D. O. (1992). Innovations in the Public Sector. Productivity \& Management Review, 16 (2), 157-171.

Hane, G. (1999) Comparing University-Industry Linkages in the United States and Japan. En Brascomb, L., Kodama, F. y Florida, R. (Eds.), University-Industry Linkages in Japan and the United States. Massachusetts: MIT Press.

Hage, J. (2005). Organizations and Innovation: Contributions from Organizational Sociology and Administrative Science. En Casper, S. 
y van Waarden, F. (Eds.), Innovation and Institutions. Northampton: Edward Elgar Publishing.

Hirschi, C. y Widmer, T. (2010). Policy Change and Policy Stasis: Comparing Swiss Foreign Policy toward South Africa (1968-94) and Iraq (1990-91). The Policy Studies Journal, 38 (3), 537-563.

Howlett, M. (1995). Policy instruments, Policy Styles and Policy Implementation: National Approaches to Theories of Instrument Choice. En Rist, R. (Ed.), Policy Evaluation. Linking Theory to Practice. Northampton: Edward Elgar Publishing Company.

. (2009). Governance Modes, Policy Regimes and operational Plans: A Multilevel-Nested Model of Policy Instrument Choice and Policy Design. Policy Sciences, 42 (1), 73-89.

Howlett, M. y Ramesh, M. (2003). Studying Public Policy. Policy Cycles and Policy Subsystems. Toronto: Oxford University Press.

Kano, S. (2005). The Innovation Agent and Its Role in UniversityIndustry Relations. En Casper, S. y van Waarden, F. (Eds.), Innovation and Institutions. Northampton: Edward Elgar Publishing.

Keeley, L. (2013). Ten Types of Innovation: The Discipline of Building Breakthroughs Hoboken: Wiley.

Kingdon, J. (1984). Agendas, Alternatives and Public Policies. Boston: Little Brown.

Klein, K. y Speer Sorra, J. (1996). The Challenge of Innovation Implementation. The Academy of Management Review, 21 (4), 10551080.

Köhler, H. y González Begega, S. (2014). Elementos para un Concepto Sociológico de Innovación. Revista de Metodología de Ciencias Sociales, 29, 67-88.

Marsh, D. y Smith, M. (2000). Understanding Policy Networks: Towards a Dialectical Approach. Political Studies, 48 (1), 4-21.

Mariñez, F. (2015). Innovación Pública. Para Que Funcionarios Públicos y Ciudadanos Actúen con Saberes Cívicos. México: Editorial Fontamara. Meeus, M. y Oerlemans, L. (2005). National Innovation Systems. En 
Casper, S. y van Waarden, F. (Eds.), Innovation and Institutions. Northampton: Edward Elgar Publishing.

Monsiváis, A., Pérez, A. y Talavera, N. (2014). Protesta Social, Política Deliberativa y Democracia: Un Análisis de los "Diálogos por la Paz" en México. Revista de Ciencia Politica, 34 (3), 623-643.

Montero, J. C. (2010). La Estrategia contra el Crimen Organizado en México: Análisis del Diseño de la Política Pública. Perfiles Latinoamericanos, 39, 7-30.

. (2013). El Concepto de Seguridad en el Nuevo Paradigma de la Normatividad Mexicana. Región y Sociedad, XXV (58), 203-238.

Moore, M. y Hartley, J. (2008). Innovations in Governance. Public Management Review, 10 (1), 3-20.

Morgan, K. (1997). The Learning Region: Institutions, Innovation and Regional Renewal. Regional Studies, 31 (5), 491-503.

North, D. (1990). Institutions, Institutional Change and Economic Performance. Cambridge: Cambridge University Press.

Ostrom, E. (2000). El Gobierno de los Bienes Comunes. La Evolución de las Instituciones de Acción Colectiva. México: UNAM-FCE.

. (2008). Collective Action and the Evolution of Social Norms. The Journal of Economic Perspectives, 14 (3), 137-158.

Ravelo, R. y Salas, A. (2006). De Paraíso a Cubil de Narcos. Proceso, 27 de marzo.

Roper, S., Du, J. y Love, J. (2008). Modelling the Innovation Value Chain. Research Policy, No. 37 (6-7), 961-977.

Sabatier, P. A. (2007). Theories of the Policy Process. Boulder: Westview Press.

Sabatier, P. A. y Jenkins-Smith, H. (Eds.). (1993). Policy Change and Learning: An Advocacy Coalition Approach. Boulder: Westview Press.

Salazar, H. (2013). Fuerza Civil. México: GENL. 
Scheel, C. (2012). El Enfoque Sistémico de la Innovación: Ventaja Competitiva de las Regiones. Estudios Gerenciales, 28, 27-39.

Scheel, C. y Pineda, L. (2015). Innovacities: Impacto de los Sistemas Regionales de Innovación en las Estrategias Competitivas de las Ciudades. Bogotá: UTADEO.

Steels, E. (2015). El Teatro del Engaño. México: Grijalbo.

Swyngedouw, E. (2005). Governance Innovation and the Citizen: The Janus Face of Governance-beyond-the-State. Urban Studies, 42 (11), 1991-2006.

Teece, D. (2010). Business Models, Business Strategy and Innovation. Long Range Planning, Vol. 43 (2-3), 172-194.

Tsebellis, G. (2006). Jugadores con Veto: Cómo Funcionan las Instituciones Políticas. México: Fondo de Cultura Económica.

Valdés, G. (2013). Historia del Narcotráfico en México. México: Debate.

Villarreal, A. y Flores, M.A. (2015). Identificación de Clusters Espaciales y su Especialización Económica en el Sector de Innovación. Región y Sociedad, XXVII, (62), 117-147.

Villarreal, T. (2009). Participación y Gestión Pública en Nuevo León, México. Enfoques, Ciencia Política y Administración Pública, VII (11), 415-437.

Recibido: 11-03-2016

Aceptación de la versión final: 31-05-2017 